\title{
Platelet Activating Factor
}

National Cancer Institute

\section{Source}

National Cancer Institute. Platelet Activating Factor. NCI Thesaurus. Code C16998.

A phosphatidylcholine derivative that modulates inflammation, vascular permeability,

allergic responses and the functions of leukocytes and platelets. 